\title{
A Comparative Review of the SAR of Amlodipine and Lercanidipine
}

\author{
Adeyomola Ismail Kazeem* \\ Pharmacist Researcher, University of Ibadan, Ibadan, Nigeria \\ *Corresponding Author: Adeyomola Ismail Kazeem, Pharmacist Researcher, University of Ibadan, Ibadan, Nigeria.
}

Received: April 26, 2019; Published: June 20, 2019

DOI: $10.31080 /$ ASPS.2019.03.0320

Keywords: Amlodipine; Lercanidipine; Hypertension; Dihydropyridine; Calcium Channel Blocker

Hypertension is one of the most important chronic diseases worldwide and is a leading risk factor for mortality [7]. Hypertension is defined as a sustained elevated blood pressure that presents as a systolic blood pressure of $\geq 140 \mathrm{mmHg}$ or a diastolic blood pressure of $\geq 90 \mathrm{mmHg}$ [5].

Antihypertensive medications are administered to reduce blood pressure and maintain the blood pressure within limits thus attaining control of blood pressure [8]. As a result of their effects, antihypertensive medications reduce mortality, morbidity and complications of hypertension [11].

In blacks generally, antihypertensive therapy is initiated with a thiazide/thiazide-like diuretic or a calcium channel blocker (CCB) as monotherapy or in combination [3]. In recent times, dihydropyridines, a class of CCB, are being prescribed more commonly in a number of eastern Asian countries. Calcium channel blockers have been shown to have no effect which increase the risk of coronary events and stroke [14].

Dihydropyridines (a class of calcium channel blockers) are one of the most commonly prescribed agent in hypertension monotherapy [9]. Studies have shown that the newer generation dihydropyridines have a relatively mild adverse effect profile and longer duration of action compared to the older generations $[2,4,10]$. They are also progressively less cardio selective with broader therapeutic spectrum than older generation dihydropyridines $[2,4,10]$.

Amlodipine is perhaps the most commonly prescribed CCB, it offers a half-life of about $36 \mathrm{~h}$, relatively higher vascular-cardiac effect ratio, lower fluid retention potential, slower onset of vascular action and relatively lower risk of adverse vascular effects than 1st and 2nd generation dihydropyridines $[13,14]$.

Lercanidipine, a highly lipophilic dihydropyridine offers relatively lower risk of adverse effects, broader therapeutic index and more stable activity. Lercanidipine shows positive potential in the management of congestive heart failure and in ischemic heart diseases [4].
The duration of action, the rate of adverse reactions, the antihypertensive effect and the pattern of protection on end organs are some of the factors that determine the clinical applications of calcium channel blockers [1].

Structure-activity relationship of amlodipine and lercanidipine: similarities, differences and consequences

This review aims to compare the structures of Lercanidipine and Amlodipine and relate the differences in their structure to the differences in their activity.
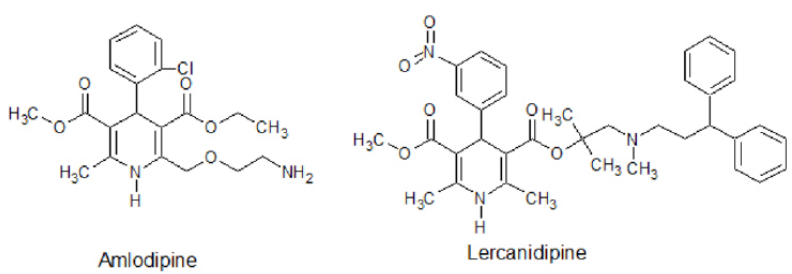

Figure

As can be seen, the C5 and C6 moieties of the dihydropyridine ring in both amlodipine are the same. While the $\mathrm{C} 6$ moiety in both drugs is methyl, recent study has shown that replacing methyl with larger groups like a phenyl group increases the activity and selectivity of dihydropydridines likely through lipophilic interactions with lipophilic spaces in calcium channels [12]. Although having larger groups at C6 improves activity by increasing lipophilic interactions, the opposite applies to $\mathrm{C} 2$ because of the steric hindrance that comes with larger moieties [12]. This is evident is the marginal difference in potency between S-amlodipine and R-amlodipine with the S-enantiomer being the more potent enantiomer. R-amlodipine has the amine substituent on C2 while S-amlodipine has its amine substituent on C6. This phenomenon is absent in lercanidipine because the moiety and $\mathrm{C} 2$ and $\mathrm{C} 6$ are identical. 
The ester groups at the $\mathrm{C} 3$ and $\mathrm{C} 5$ are required for binding to glutamate residues in calcium channels by coordination through a calcium ion bridge [12]. The 2 oxygen atoms of the ester groups are required to chelate 2 calcium ions for coordination to glutamate residues to occur. Replacing the ester groups with carbonyls like ketones that offer one oxygen atom or functional groups that will not chelate calcium ion reduces activity of dihydropyridines markedly [12]. The C3 and C5 moieties also determine enantiomeric selectivity [12] as seen in the relatively higher activities of S-amlodipine and S-lercanidipine compared to their enantiomers.

Also, in lercanidpine, the C3 substituent offers significant bulkiness which raises the lipophilicity of lercanidipine over that of amlodipine. Studies show that the lipophilicity of dihydropyridines may be linked to reduced adverse effect, reduced cardioselectivity and longer duration of action [4]. Hence, the relatively higher tolerability and reduced cardioselectivity observed with the use of lercanidipine compared to amlodipine.

At the $\mathrm{C} 4$, the phenyl group offers increased activity relative to alkyl and cycloalkyl groups. The phenyl group interacts with tyrosine residues in calcium channels through a ring-to-ring interaction [12]. Substitution on the phenyl group with an electron-withdrawing substituent is also essential for activity with the ortho and meta positions more preferred to the para position [12].

This substitution is necessary to maintain a fixed configuration which keeps the phenyl group at C4 perpendicular to the dihydropyridine ring - a configuration essential for activity [6,12]. While amlodipine contains a chlorine atom in the ortho position at the C4 phenyl ring, lercanidipine contains a nitro at the meta position. While these two substituents both offer the electron-withdrawing effect necessary for the aforementioned perpendicular configuration, the nitro substitution of lercanidipine will have the relatively superior effect thus the more stable configuration.

The hydrogen atom from the $\mathrm{N}-\mathrm{H}$ and the substitution on $\mathrm{C} 4$ determine the degree of affinity to the calcium channels [12]. The hydrogen atom takes part in hydrogen bonding with the receptors and the effect is similar in amlodipine and lercanidipine [12]. However, the C4 substituent in lercanidipine is more stable and this has a higher affinity for calcium channels than amlodipine.

\section{Bibliography}

1. Angela L Wang., et al. "New generations of dihydropyridines for treatment of hypertension". Journal of Geriatric Cardiology 14.1 (2017): 67-72.
2. Aouam K and Berdeaux A. "Dihydropyridines from the first to the fourth generation: better effects and safety". Therapie 58.4 (2003): 333-339.

3. Armstrong C and Joint National C. "JNC8 guidelines for the management of hypertension in adults". American Family Physician 90.7 (2014): 503-504.

4. Chandra KS and Ramesh G. "The fourth-generation Calcium channel blocker: cilnidipine". Indian Heart Journal 65.6 (2013): 691-695.

5. Chobanian AV., et al. "Seventh report of the Joint National Committee on Prevention, Detection, Evaluation, and Treatment of High Blood Pressure". Hypertension 42.6 (2003):1206-1252.

6. D Hadjipavlou-Litina. "Calcium ion channels and their blockers". In Ion Channels and Their Inhibitors, S. P. Gupta, Ed., Springer, New York, NY, USA (2011): 265-288.

7. Ezzati M., et al. "Comparative Risk Assessment Collaborating Group Selected major risk factors and global and regional burden of disease". Lancet 360 (2002): 1347-1360.

8. Jarari N., et al. "A review on prescribing patterns of antihypertensive drugs". Clinical Hypertension 22 (2015): 7.

9. Kjeldsen SE., et al. "Clinical implications of the 2013 ESH/ESC hypertension guidelines: targets, choice of therapy, and blood pressure monitoring". Drugs in R and D 14.2 (2014): 31-43.

10. Malhotra HS and Plosker GL. "Barnidipine”. Drugs 61.7 (2001): 989-996.

11. McVeigh GE1., et al. "Goals of antihypertensive therapy". Drugs 49.2 (1995): 161-175.

12. Moataz A., et al. "1,4-Dihydropyridine Calcium Channel Blockers: Homology Modeling of the Receptor and Assessment of Structure Activity Relationship". International Scholarly Research Notices (2014): Article ID 203518.

13. Toal CB., et al. "Long-acting dihydropyridine calcium-channel blockers and sympathetic nervous system activity in hypertension: a literature review comparing amlodipine and nifedipine GITS”. Blood Press 21.1 (2012): S3-S10.

14. Wang JG., et al. "Use of dihydropyridine calcium channel blockers in the management of hypertension in Eastern Asians: a scientific statement from the Asian Pacific Heart Association". Hypertension Research 34.4 (2011): 423-430.

Volume 3 Issue 7 July 2019

(C) All rights are reserved by Adeyomola Ismail Kazeem. 